\title{
DES CONDITIONS JURIDIQUES NECESSAIRES A LA MODERNISATION ET A L'EXTENSION DU BARRAGE HYDRAULIQUE D'INGA.
}

\author{
Par Banza Ilunga Aimé
}

\section{INTRODUCTION}

La RDC reste l'un des rares pays au monde doté d'importantes ressources hydroélectriques. Son potentiel énergétique exploitable pour l'hydroélectricité est évalué à $774000 \mathrm{GW}$ h, soit $66 \%$ du potentiel de l'Afrique Centrale, $35 \%$ du potentiel global du continent africain et $8 \%$ du potentiel de production annuelle mondiale. La RDC vient en effet, en première place en Afrique Centrale (avant le Cameroun, 115000 GW h). Dans le monde, elle se place en troisième position après la Chine et le Canada ${ }^{2}$.

Le potentiel de la RDC se traduit par une puissance exploitable d'environ $100000 \mathrm{MW}$ dont près de la moitié (44000 MW) est concentrée sur le seul site d'INGA, ce qui fait de ce dernier le plus grand gisement d'énergie hydraulique au monde. Il est installé sur le majestueux fleuve Congo, long de $4700 \mathrm{~km}$ avec un débit de $40000 \mathrm{~m}^{3} / \mathrm{s}$ en moyenne et un bassin hydrographique qui constitue un grand axe du système de communication du pays.

Ainsi, le présent travail porte dans une large mesure sur ce barrage hydraulique d'Inga au regard de son projet de modernisation envisagée par le gouvernement congolais. S'agit-il en clair, de dégager les conditions juridiques nécessaires à la modernisation et à l'extension de ce barrage bien que les défis à la fois politiques, techniques, financiers, économiques qu'environnementaux sont importants. Aussi où glaner le fonds nécessaire pour opérer cet épanouissent « modernisation » du site d'Inga? Qui est légalement responsable pour les travaux de modernisation selon la loi sur le secteur de l'électricité? Mais il convient de savoir encore, quels aspects juridiques doit-on respecter pour agrandir les barrages d'Inga? Par exemple, la garantie d'alimentation en courant de la population face à l'intérêt économique d'exporter l'électricité vers l'étranger. Et comment la loi sur l'électricité règle-t-elle ces aspects alors qu'il n'y a pas encore un règlement pris en rapport avec cette loi.

Pour répondre à ces préoccupations, commençons par présenter le site d'Inga luimême : ce site comprend le dispositif déjà existant (Inga I et II). Ces deux barrages sont situés en RDC sur le fleuve Congo dans la province du Bas-Congo, à proximité de la ville de Matadi. Repris de projets belges lancés à l'époque coloniale et construits avec une aideinternationale. Les barrages d'Inga I, entré en fonction en 1972, avec une capacité de 351

1 Assistant à la Faculté de Droit de l'Université de Lubumbashi.Mail: Aimebanza25@yahoo.fr

2 KASEMUANA Séraphin, Rapport : Energie et Ecodéveloppement en RDC, Hélio International, Observatoire de la viabilité énergétique, 2006. 
MW et Inga II qui fonctionne depuis 1982, d'une capacité installée de 1424 MW; sont sousutilisés (environ $20 \%$ de leurs capacités) faute d'entretien.

Le développement d'Inga III et du Grand Inga ne sont actuellement qu'au stage de projet. Ce sont donc des projets gigantesques à dimension continentale et même mondiale. ${ }^{3}$

Par ailleurs, l'Etat congolais ne peut à lui seul répondre efficacement aux enjeux énergétiques, en raison de l'insuffisance des ressources financières du gouvernement qui doit faire face à de nombreuses priorités. L'insuffisance des initiatives privées dans le secteur énergétique et le manque de financement sont quelques uns des obstacles au relèvement du secteur de l'électricité en $\mathrm{RDC}^{4}$.

Sachant que la modernisation du site d'Inga demande des moyens financiers colossaux, les conditions juridiques nécessaires à cette modernisation s'exprimeraient par la libéralisation du secteur d'électricité et par des règles de partenariat public-privé (partenaires nationaux ou internationaux) et d'accès au financement, par des traités et accords internationaux. Il faut mobiliser d'importantes ressources financières et passer par des projets à vocation régionale ou internationale c'est-à-dire qui impliquent plusieurs Etats et aussi trouver une part des ressources à l'intérieur du pays.

Ainsi, avec Grand Inga, l'intérêt des bailleurs internationaux et des investisseurs privés se confirme pour les ressources électriques et il semble que l'on se dirige vers la création d'un marché global de l'électricité en Afrique. Grand Inga est le plus important projet hydroélectrique dans le monde. Ce gigantesque barrage, est partie intégrante d'une vision plus grande de la communauté économique internationale de développer la capacité énergétique à travers l'Afrique afin de stimuler le développement économique et industriel du continent. Grand Inga pourrait produire jusqu'à 40000 MW d'électricité, soit deux fois plus d'énergie que le barrage des Trois-Georges en Chine, et plus de tiers de l'électricité totale actuelle sur tout le continent africain. Cela nécessiterait 80 milliards de dollars estimés. Bien que les études de faisabilité sur Grand Inga ne sont pas encore totalement complètes, le projet est déjà présenté comme le moyen pour « illuminer l'Afrique » tant par les gouvernements qui espèrent recevoir de l'énergie de ce projet que par les compagnies qui cherchent à en tirer profit. Grand Inga est listé comme projet prioritaire de la Communauté de développement de l'Afrique Australe (SADC), le Nouveau Partenariat pour le Développement de l'Afrique (NEPAD) et le Conseil Mondial de l'Energie (CME).

Ainsi, il est prévu de développer Inga 3 (4800 MW) comme $1^{\text {ère }}$ phase de Grand Inga pour exporter l'énergie vers l'Afrique du Sud et d'autres pays voisins et aussi attirer les industries à forte consommation d'énergie en RDC. A ce sujet, le $1^{\text {er }}$ Avril 2014, le Conseil d'administration de la Banque mondiale a approuvé le projet Inga III pour un montant de 12 milliards de dollars.

3 TOUROT Nicolas, Les Projets de développement hydroélectrique en Afrique Noire, Mémoire de Maîtrise, Paris I, 2003, p7.

4 YUMA MULIMBI Albert, Le Rôle du Secteur Privé Congolais dans la Reconstruction de l'Etat et la Réalisation d'un Développement économique durable en RDC, In Conférence de la FEC, Bruxelles,2008, p28. . 
Les barrages existants Inga I et II connaissent une réhabilitation avec l'assistance financière de la Banque Mondiale, de la Banque européenne d'investissement et de la BAD. Inga II est également soumis à une réhabilitation à travers un plan de privatisation partielle avec une compagnie dénommée « MagEnergy » ainsi qu'avec le soutien financier de la South Africas 's Industrial développement corporation. ${ }^{5}$

Cependant, le Gouvernement congolais doit être clairvoyant et incrédule et ne pas céder totalement au dictat des institutions financières internationales (IFI) et des multinationales, parce qu'en réalité il pourrait s'agir d'une électricité qui n'éclairera pas les congolais ou les africains. Ainsi, si l'idée avancée est de répondre aux besoins d'un continent sous-électrifié, on peut néanmoins s'inquiéter des effets de ces mégaprojets et du modèle de développement qui le porte c'est-à-dire orienté sur la réponse aux besoins économiques privés. Si les investisseurs se ruent sur ces projets, c'est d'abord pour bénéficier d'une énergie abondante et bon marché. La satisfaction des besoins des congolais pourtant sous-desservis avec $9 \%$ de desserte n'est pas une priorité. Les besoins financiers pour l'électrification locale devraient donc être inclus au budget de ces projets.

Il faudrait donc que dans les traités et les accords de partenariat que la RDC signe avec ses partenaires, qu'il y ait des dispositions pertinentes qui tiennent compte de la garantie d'alimentation en électricité de la population face à l'intérêt d'exporter l'électricité vers l'étranger. A mon avis, l'exportation ne pourrait concerner que le surplus d'énergie électrique disponible. D'abord desservir la population.

A ce sujet, la loi de novembre 2013 sur le secteur d'électricité consacre la libéralisation du secteur en ce qui concerne la production, le transport et la distribution de l'électricité. Une libéralisation que nous vous voulons tempérée c'est-à-dire celle qui tient compte des besoins de rentabilité des investisseurs mais aussi des besoins d'alimentation à bas prix de la population qui a au départ un pouvoir d'achat faible. Mais pour éviter les conséquences d'un libéralisme excessif des opérateurs mercantiles, la loi sus visée envisage des conditions de concurrence et des règles et des modalités de fixation des tarifs de l'électricité. Ces tarifs sont fixés suivant les principes de vérité, d'égalité, d'équité et de non transferabilité des charges. Celles-ci étant auditables. Ils sont fixés sur proposition de l'Autorité de Régulation, par Arrêté interministériel des Ministres ayant l'Economie et l'Electricité dans leurs attributions. Ces tarifs sont publiés au Journal Officiel par l'Autorité de Régulation. ${ }^{6}$ Comme on le voit, il n'y a que les règlements (arrêtés ministériels) qui seront pris qui conféreraient à cette loi son application effective. Précisons que cette loi est déjà adoptée par les deux chambres du parlement depuis le 15 novembre 2013 et n'attend à ce jour que sa promulgation par le Président de la république pour son entrée en vigueur.

5 Agence Française de développement sur le projet du barrage d'Inga III, RDC/AFD, Kinshasa, juin 2012, p3.

6 Article 21 de la Loi relative au secteur de l'électricité en RDC, cabinet du Premier Ministre, Kinshasa, Novembre 2013. 
Quant aux défis juridiques que autres, les risques que font peser ces projets de développement titanesques sont sérieux : déplacement des populations, destruction des écosystèmes fluviaux mais aussi la corruption liée à l'opacité des contrats, l'exécution de ceux-ci et appellent à une vigilance accrue. Les traités et accords de partenariat y relatifs doivent amplement en tenir compte. Comme, le Traité de coopération énergétique signé le 30 octobre 2013 à Kinshasa entre la RDC et la République Sud Africaine, où sont contenues des conditions pertinentes de modernisation du Grand Inga.

On le sait, les communautés déplacées d'Inga se battent depuis 1970 pour obtenir des compensations justes, mais n'ont rien reçu jusqu'ici. Le mauvais entretien ainsi que les problèmes financiers qu'ont connu Inga I et Inga II soulèvent des questions sur les risques qui pourraient survenir avec Inga 3 et Grand Inga. C'est la question de responsabilité se pose.

A ces causes, le gouvernement congolais avec ses partenaires sont appelés à une vigilance accrue en mettant sur pied des conditions juridiques équitables pour le développement du complexe d'Inga.

\section{CHAPITRE I. DE LA MISE SUR PIED DU PROJET INGA}

D'une manière succincte, nous présentons dans cette partie les grandes étapes qui ont conduit à la mise en route du barrage d'Inga.

Les barrages hydroélectriques d'Inga sont deux barrages situés en RDC sur le fleuve Congo dans la province du Bas-Congo, à proximité de Matadi ${ }^{7}$. Repris des projets belges lancés à l'époque coloniale et construits avec une aide internationale, ces deux ouvrages distincts sont dénommés Inga I et Inga II. Le développement du Grand Inga et sa 1ere phase d'Inga III sont encore en projet. Ces ouvrages à l'instar de l'usine sidérurgique de Maluku sont construits dans le cadre d'une politique de prestige du pays par le régime de Président Mobutu. La maintenance des ouvrages n'étant pas assurée, ils fonctionnent à capacité réduite. Ils sont exploités par la SNEL.

Comme d'autres ouvrages pharaoniques créés dans le pays au service des politiques de prestige qui ne purent être assumés, ils sont qualifiés d'éléphants blancs.

Ainsi, la nette appréhension de l'histoire du barrage Inga ne peut passer que par une analyse par étape.

\section{SECTION I. DES ETUDES DE FAISABILITE}

En mai 1957, il a été crée un Comité International composé d'universitaires belges et d'ingénieurs Conseils étrangers et chargé d'étudier les conclusions remises par les bureaux d'études en vue de faire des propositions concrètes sur le projet d'Inga au gouvernement

7 VAN DEUREN Pierre, Aménagement du Bas-Congo, Projet, Bruxelles, 1928, p. 12. 
belge. Les conclusions de ce Comité ne se sont limitées qu'à démontrer la faisabilité du projet dans sa dimension technique. ${ }^{8}$

L'Institut d'Inga fut crée par Arrêté Royal en décembre 1957. Il avait pour mission d'étudier, en faisant éventuellement appel à des organismes publics ou privés spécialisés, l'ensemble de l'équipement hydroélectrique du site d'Inga, le mode de financement des travaux et les possibilités d'utilisation du courant électrique.

En 1959, l'Etablissement public d'Inga fut crée en qualité de maître d'œuvre, qui allait être chargé de construire et d'exploiter les centrales. Les contradictions entre les deux instituts ont conduit à la perte de l'institut d'Inga. Après l'indépendance du Congo en 1960, la Belgique s'est vue débarquée du projet au profit des Italiens.

En 1963, fût créée la société italo-congolaise de développement industriel (SICAI), laquelle va signer un Protocole d'accord avec le gouvernement congolais représenté par le haut commissaire de l'Etablissement Public d'Inga. Ce protocole affirmait « le désir du gouvernement congolais de passer dans un bref délai à la première phase de la mise en valeur d'Inga » et confirmait à SICAI la mission d'étudier les conditions économiques requises pour la réalisation de la centrale d'une puissance de $300 \mathrm{MW}$.

Le rapport d'une mission commandée par SICA I en 1964, présente les conclusions suivantes « en analysant (les problèmes du couplage d'Inga avec une usine aluminière) sous l'angle de l'économie générale du pays, il ressort clairement que les bénéfices prévisibles directs et indirects ne sont pas proportionnels aux investissements nécessaires et qu'il serait beaucoup plus intéressant pour le Congo d'affecter le montant de ces investissements à des installations industrielles produisant pour le marché intérieur $»^{9}$.

Le rapport SICAI vient ainsi à substituer à l'installation d'industries fortement consommatrices d'énergie, l'implantation d'une usine sidérurgique. Il a tablé sur l'existence de minerai de fer au Katanga.

Ce rapport a eu gain de cause auprès des Institutions internationales de l'époque notamment la Commission Economique pour l'Afrique des nations Unies (CEA) et le Fonds Spécial de Nations Unies.

Initialement prévu à $300 \mathrm{MW}$, le plan financier de la centrale a dû subir une restriction. La centrale devait démarrer avec 3 groupes de $50 \mathrm{MW}$ chacun, avec la possibilité d'augmenter le nombre des groupes en fonction des besoins réels.

Après la première visite de Mr. MOBUTU aux USA, il remet en question les dernières hypothèses, il décide de démarrer la centrale avec 6 groupes et non 3 comme prévu sans aucune justification. En outre, il commanda la construction d'une ligne électrique à haute tension d'Inga à Moanda en prévision de l'implantation d'un port en eau profonde dans cette localité. Ildemanda en même temps le début des travaux de la centrale Inga II.

8 MUKADI José, Historique de la mise en route du barrage d'Inga, 21 décembre 2006,in www.google.fr (15/3/2013).

9 MUKADI José, op cit, p. 5. 
Et donc, on constate que s'il est vrai que sur le plan technique, le site d'Inga réunissait des conditions exceptionnelles, mais sur le plan juridico- économique, les avantages étaient moins évidents. Malgré les moult études réalisées pendant plusieurs années par de grands bureaux d'études du monde, aucune d'elles n'a pu démonter la viabilité économique qui pouvait motiver la mise sur pied du barrage d'Inga.

\section{SECTION 2. DE L'EXECUTION DES TRAVAUX}

SICAI et EDF (Electricité de France) ont été retenus comme ingénieurs conseils, respectivement pour Inga I et Inga II, afin de suivre les travaux pour le compte de la RDC.

Ainsi, en 1972, le barrage I sera mis en fonction avec une capacité de 351 MW et dix ans plus tard donc en 1982, Inga II entrait en service avec une capacité de 1424 MW. Mais tous deux fonctionnent actuellement à $20 \%$ de leur capacité, la plupart des turbines étant à l'arrêt faute de pièces de rechange et d'entretien.

A l'heure actuelle, les réseaux d'évacuation associés aux centrales existantes Inga I et II sont constitués par des lignes à haute et très haute tension orientés suivant trois axes, à savoir :

- L'axe Inga- Kinshasa/Bandundu -Brazzaville

- L'axe Inga-Matadi/Boma au Bas-Congo

- L'axe Inga -Katanga-Afrique Australe

De ces trois axes, la voie Inga-Katanga-Afrique Australe est celle qui pour l'instant, a vocation de se muer en autoroute de l'énergie moyennant certains aménagements de modernisation. En effet, cet axe comporte déjà une infrastructure importante, en l'occurrence la ligne à courant continu bipolaire $500 \mathrm{KV}$, Inga-Kolwezi, longue de $1774 \mathrm{Km}$ et dimensionnée pour un transit de $1120 \mathrm{MW}$. L'énergie d'Inga est livrée présentement à ZESA (Zimbabwe) et Eskom (RSA) à l'occurrence de $100 \mathrm{MW}$ et $110 \mathrm{MW}$. La ligne d'interconnexion $220 \mathrm{KV}$ Karavia (RDC)- Luano (Zambie) qui est un maillon de l'axe Inga-Katanga-Afrique Australe a une limite thermique de $340 \mathrm{MW}$ et nécessite d'être modernisée. Il convient de noter que le réseau en aval de Kolwezi est en courant alternatif. Le couloir RDC-RSA, long de $3676 \mathrm{~km}$ au total, passe par les principaux postes suivants : Inga -Kolwezi -Karavia (RDC)LUANO (Zambie)-Kariba sud (Zimbabwe) -ALASKA -SHERWOOD-INSUKAMIN IMATIMBA (RSA). ${ }^{10}$

\section{SECTION 3. DE LA RENTABILITE}

Depuis la mise en service des barrages d'Inga la consommation de l'énergie au niveau national n'a jamais dépassé $25 \%$ de sa puissance totale installée.

L'usine sidérurgique qui a justifié la construction d'Inga, était un fiasco et aussi considéré comme éléphant blanc. Quant à l'électrification du pays, malgré ce potentiel énorme, 
le taux de desserte nationale en électricité est un de plus bas du monde : soit $9 \%$ (en zone urbaine que rurale).

La SNEL, l'entreprise qui a en charge la production, le transport et la distribution de l'électricité, est aujourd'hui confrontée aux multiples problèmes de mégestion, de financement et même de recouvrement mensuel des factures d'électricité.

Constatons qu'Inga n'a pas encore été rentable pour la RDC, comme un moyen de développement du pays comme souhaité. Il n'a servi qu'à endetter lourdement le pays au profit des politiciens et bailleurs de fonds internationaux et les industries occidentales.

Rappelons qu'entre 1973 et 1979, la dette congolaise a été multipliée par 4, passant de 1,4 milliard à 4,5 milliard de dollars. Ceci étant dû aux coûts élevés de ces projets "éléphants blancs » et aux conséquences de la zaïrianisation et ses revers de rétrocessions anarchiques ${ }^{11}$. Loin d'améliorer les conditions de la population congolaise, ces investissements n'ont fait qu'enfoncer les congolais dans la pauvreté. Alors que seulement $9 \%$ de la population est desservie en électricité (non fiable), tout le peuple congolais doit supporter le poids de la dette. Pis encore, le remboursement de cette dette s'est accompagné de l'application de fameux plans d'ajustement structurel dictés par la Banque Mondiale et le FMI, suite à l'éclatement de la crise de la dette du tiers-monde en 1982 et comme conséquences : la part du budget destinée à l'enseignement est passé brutalement de $25 \%$ à $3 \%$, des milliers de travailleurs des entreprises publiques ont été licenciés, les salaires des fonctionnaires ont été gelés... La priorité de était le remboursement de la dette, accaparant ainsi des ressources vitales pour le développement de la population : jusqu'à $50 \%$ du budget du Zaïre durant les années 1983 à 1989.

Les autorités actuelles n'ont qu'à être clairvoyantes en initiant des projets de modernisation d'Inga, qui aient d'impact social palpable sur la population congolaise. En d'autres termes, qu'ils soient rentables pour le pays. C'est pourquoi, il y a nécessité des conditions juridiques commutatives y relatives.

\section{CHAPITRE II. DES CONDITIONS JURIDIQUES NECESSAIRES A LA MODERNISATION ET A L'EXTENSION D'INGA}

Pour moderniser les installations d'Inga, en tenant compte de dernières évolutions techniques, il n'est pas facile aujourd'hui de faire une symbiose dans la réhabilitation d'Inga entre une technologie des années 70 et une technologie de pointe actuelle. Ainsi, cette modernisation et extension demandent de gros moyens financiers et investissements que seule la RDC ne peut mobiliser d'où le recours aux partenaires nationaux et internationaux, publics ou privés, bailleurs de fonds, entreprises nationales ou multinationales.

C'est pourquoi cette modernisation ne peut aller qu'avec la définition des conditions juridiques idoines : sur le plan interne, doter la RDC d'une loi moderne, cohérente et attrac-

11 LUKOMBE NGHENDA, Zaïrianisation, Radicalisation et Rétrocession en République du Zä̈re, Considérations Juridiques, éd. PUZ, Kinshasa, 1979, p. 377. 
tive dans le secteur d'électricité; d'une législation économique compétitive et attractive; l'assainissement du climat des affaires; créer des conditions favorables au partenariat public-privé et d'accès au financement et aussi sur le plan externe, créer des conditions juridiques découlant des conventions internationales, puisque l'exploitation de l'énergie produite par Inga fait naître un élément d'extranéité.

\section{SECTION 1. DE LA NECESSITE D’UN CODE DE L'ELECTRICITE EN RDC}

Les conditions juridiques de modernisation et d'extension du barrage Inga sont dans une mesure liées à l'existence d'une loi sur le secteur d'électricité en RDC. Laquelle loi est un préalable à toute reforme dans ce secteur. Cela est autant vrai qu'on ne peut parler d'énergie électrique en RDC sans faire allusion au barrage Inga. Ainsi, à ces jours, parler de principales faiblesses du secteur de l'électricité en RDC, c'est faire allusion : - au cadre juridique et institutionnel inadapté par rapport à l'évolution de l'environnement politique, économique, technologique et social au plan national et international; les taux d'accès aux services d'électricité les plus faibles du continents; la vétusté des infrastructures électriques et principalement celles d'Inga; l'électrification rurale embryonnaire ${ }^{12}$.

En effet, l'absence d'un cadre juridique clair, attrayant et sécurisant dans le secteur de l'électricité depuis l'accès de notre pays à l'indépendance, aggravé par le manque d'anticipation et de planification explique en grande partie la situation dramatique du contraste que vit notre pays, à savoir : d'énormes potentialités hydroélectriques estimés à $100.000 \mathrm{MW}$ dont 44.000 MW au seul site d'Inga, avec un faible taux d'accès des populations à l'électricité.

Dans le souci de doter la RDC d'un cadre juridique adéquat dans le secteur de l'électricité et pour lancer les jalons des solutions durables pour le développement de l'électricité du pays et à son développement économique, le gouvernement a choisi la voie de la libéralisation du secteur, perspectives du projet de loi de novembre 2013 relative au secteur de l'électricité.

Avec au total à ce jour 125 articles en dix titres (subdivisé en chapitres, sections et paragraphes) précédés d'un exposé des motifs, ce projet de loi affirme en substance ${ }^{13}$ :

- La libéralisation du secteur et l'ouverture du marché de l'électricité;

- Le principe de la décentralisation et de la répartition des compétences entre le Gouvernement centrale et les entités décentralisés;

- La déclaration de tout site hydroélectrique ou géothermique en site d'utilité publique;

- Le principe de la protection de l'environnement pour tous les projets de développement de secteur;

12 Revue du DSCRP, RDC, Kinshasa, mars 2010, p. 2.

13 Loi relative au secteur de l'électricité en RDC, cabinet du Premier Ministre, Kinshasa, Novembre 2013. 
- L'obligation pour le gouvernement de promouvoir l'électrification rurale, périurbaine et des agglomérations ou villes secondaires afin d'accroitre le taux de desserte;

- La garantie de la protection tant de l'opérateur que du consommateur, habilités tous à saisir l'Autorité de Régulation afin de régler tout litige surgissant dans leurs rapports contractuels.

Dans la même occurrence, nous pouvons citer les innovations importantes dans le cadre de cette loi :

- Le régime juridique des activités distinctes, l'exercice des activités dans le secteur est soumis à un des régimes suivants : la concession, la licence, l'autorisation, la déclaration ou la liberté;

- La fixation des principes de tarification de l'électricité : équité, égalité, vérité... en vue de protéger le consommateur;

- La création d'un nouveau cadre institutionnel composé en outre du Ministre en charge du secteur d'électricité; de l'Autorité de Régulation dont la mission est notamment de promouvoir la libre concurrence et le contrôle des activités des opérateurs du secteur; de l'Agence d'Electrification Nationale et du Fonds National d'Electrification comme instrument de financement des projets d'électrification;

- L'instauration d'un mécanisme de règlement des différends entre opérateurs et entre ceux-ci et consommateurs;

- La répression de certains comportements jusque-là non infractionnels pour promouvoir l'efficacité énergétique et la gestion rationalisée et plus responsable de l'énergie disponible. Il s'agit des raccordements frauduleux, de la fraude d'électricité, du recel de matériels électriques et des réseaux, de l'entrave à l'exercice de la mission de contrôle et la construction sur ou sous les lignes électriques.

En clair, de l'avènement et application effective de ce code d'électricité se dégageront des conditions juridiques nécessaires à l'épanouissement du site Inga. Ce code qui consacre principalement la libéralisation du secteur, ouvre la porte aux investisseurs nationaux et étrangers dans ce secteur, notamment dans la production, la distribution et l'exportation de l'électricité. Il est donc évident que ces opérateurs concentrent leurs investissements à la réhabilitation mieux à la modernisation et extension d'Inga, qui reste le principal gisement électrique de la RDC, voire de l'Afrique et du monde.

\section{SECTION 2. DES CONDITIONS JURIDIQUES LIEES AUX CONVENTIONS INTERNATIONALES ET AU PARTENARIAT PUBLIC-PRIVE}

Le site d'Inga offre l'avantage de pouvoir construire plusieurs barrages étape par étape et sans beaucoup de travaux de génie civil. Il est l'un des projets intégrateurs d'Afrique; capable de relier les pays de l'Afrique Australe à ceux de l'Afrique du Nord. Dans sa phase 
finale, le site d'Inga sera capable de fournir de l'énergie électrique aux pays de l'Europe Méditerranéenne ${ }^{14}$.

Ainsi, les conditions juridiques favorables à la modernisation et l'extension de ce potentiel mondial ne peuvent s'exprimer que par des règles de partenariats internationaux, bilatéraux ou multilatéraux, sur des contrats bilatéraux ou multilatéraux et sur des conventions et accords sur le développement du site d'Inga, entre la RDC et divers bailleurs de fonds, divers Etats et transnationales incités à œuvrer dans le secteur.

Cependant, si ces partenariats ou contrats se révèlent entachés des conditions (clauses) léonines, ils ne serviront qu'à creuser de nouveau la dette congolaise que nous qualifierons de « dette illégitime ».

Parlons alors de ces projets de modernisation et d'extension d'Inga.

\section{§ 1. DES PROJETS DE MODERNISATION ET D'EXTENSION DU COMPLEXE INGA}

\section{$1^{\circ}$. DU DIAGNOSTIC}

Relevons avec détails qu'en termes de diagnostic dans le secteur de l'électricité congolaise, on cite notamment le manque des services adéquats découlant d'un état de dégradation très avancée des installations d'Inga : installations de production, transport de distribution et d'exportation d'électricité, confiées à la SNEL, cela s'accompagne notamment par l'immobilisation de près de la moitié des groupes installés dans les centrales interconnectées et d'autres dans les centrales isolées. Cette situation peut s'expliquer également par la précarité des ouvrages de génie civil et équipement électromécaniques, la saturation des réseaux de transport et de distribution de l'électricité ainsi que la vétusté et l'obsolescence de l'appareillage haute tension et des surcharges des équipements favorisant leur vieillissement précoce $^{15}$.

Face à ce diagnostic, les remèdes passent par la réalisation entre le gouvernement et ses bailleurs, des investissements visant aussi bien la fiabilisation, la réhabilitation des infrastructures existantes que la construction de nouvelles infrastructures en vue d'assurer d'une part l'électrification des centres urbains et ruraux du pays et d'autre part l'exportation du surplus d'énergie électrique vers d'autres pays.

C'est pourquoi la modernisation et l'extension du complexe d'Inga s'avèrent un remède indispensable.

14 RUDO SANYANGA, "Barrage Grand Inga, $R D C$ », in International Rivers, Berkeley, USA, mars 2012, p6.

15 KINTAMBU KIBANIMBA, Estimation des besoins et coûts dans le secteur de l'énergie électrique pour l'atteinte des OMD dans le cadre d'une planification axée sur les résultats, Mémoire, Economie et Finance, UNIKIN, Kinshasa, 2009, p. 28. 


\section{$2^{\circ}$. DE LA MODERNISATION D'INGA IET INGA II}

La RDC a élaboré depuis 2001 un ambitieux plan de modernisation du barrage Inga, pour exporter de l'énergie jusqu'en Afrique de l'ouest et faire du secteur l'une des sources principales des ressources nationales devant le diamant et le cuivre ${ }^{16}$.

\section{Recours aux partenaires}

Le gouvernement, avec des dettes contractées auprès des IFI (Institutions Finances Internationales) est en train de réaliser des investissements prioritaires pour moderniser les barrages I et II d'Inga.

Le Projet de Développement des Marchés d'Electricité pour la consommation domestique et l'exportation (PMEDE) et le Projet du Marché de l'électricité en Afrique Australe (SAPMP) d'un montant de 900 millions de dollars ont été mis en place par la Banque Mondiale; la Banque Européenne d'investissement et la Banque Africaine de développement. Ces projets portent sur la réhabilitation des centrales d'Inga I et II, la rénovation et l'expansion du système de distribution de Kinshasa et de sa banlieue, la construction d'une nouvelle ligne HTCA $220 \mathrm{KV}$ entre Inga et Kinshasa, la réhabilitation et la rénovation de la ligne HTCC $500 \mathrm{KV}$ entre Inga et Kolwezi (1774 km). ${ }^{17}$

Dans le PMEDE, il est prévu la réhabilitation des installations hydroélectriques d'Inga, notamment la rénovation des turbines et autres installations pour améliorer la capacité opérationnelle et la fiabilité des centrales d'Inga I et II. Selon les données disponibles, il faut passer d'une production de $700 \mathrm{MW}$ à environ $1300 \mathrm{MW}$. Il faut réhabiliter 8 turbines sur les 12 existantes sur le site d'Inga. Quant au SAMP, il vise la facilitation du développement d'un marché d'électricité efficace dans la SADC.

Par ailleurs, située au centre de l'Afrique, entourée de 9 pays, la RDC occupe une position stratégique qui la place dans trois pools énergétiques faisant partie intégrante des organisations régionales les trois pools sont : - le SAPP (South Africa Power Pool) qui dérive de la SADC; le EAPP (East Africa Power Pool) qui dérive des organisations des Etats de l'Afrique de l'Est et le PEAC (Pool des Etats de l'Afrique Centrale) dérivant de la communauté économique des Etats d'Afrique Centrale.

Ainsi, cette position de la RDC dans ces trois pools oblige l'exportation de l'excèdent d'électricité. Le Projet SAMP augmentera la disponibilité et la fiabilité de l'électricité à faibles coûts et respectueuse de l'environnement au sein du Pool énergétique d'Afrique Australe (SAPP) en plus de soutenir la meilleure intégration et davantage d'échanges commerciaux entre les pays du SAPP.

Le SAPP connecte les réseaux électriques de 12 pays dont 10 pays de la sous-région d'Afrique Australe (Angola, Botswana, Lesotho, Malawi, Mozambique, Namibie, RSA,

16 Ministre de 1'Energie, Rapport, Colloque annuel des Ministres de l'énergie de la SADC, Kinshasa, juin 2001.

17 Revue du DSCRP, op.cit, p.4. 
Swaziland, Zambie); la Tanzanie et la RDC. Neuf de ces pays sont membres opérationnels c'est-à-dire qu'ils sont reliés au réseau qui transporte environ $97 \%$ de l'énergie produite par le SAPP.

Le conseil d'administration de la Banque mondiale a approuvé le 28 juin 2012 un financement supplémentaire sous la forme d'une subvention de l'Association Internationale de Développement (IDA) d'un montant de 201,5 millions de dollars, en vue de renforcer la capacité de transport de la RDC afin de mieux répondre à la demande nationale en énergie et de soutenir l'intégration de ce pays au marché régionale de l'énergie en Afrique Australe. Ce financement supplémentaire sera utilisé pour mettre en état la longue ligne de transport de $2300 \mathrm{Km}$ reliant le site d'Inga à la Zambie.

C'est pourquoi la Banque Mondiale a commencé aujourd'hui à libérer progressivement les fonds nécessaires à ces deux projets susvisés (PMEDE et SAPMP). Et, Eustache Ouayoro, alors directeur des opérations de la Banque Mondiale en RDC, affirme qu'un des éléments stratégiques de la stratégie d'Aide-pays est « la remise en état et l'expansion des systèmes de transport d'électricité et le soutien du rôle de la RDC au sein du développement des réseaux régionaux d'énergie afin d'obtenir une croissance économique robuste, durable et partagée $»^{18}$.

\section{Recours aux ressources propres de la RDC}

Toutefois, pour procéder aux réhabilitations et modernisation du site Inga, et principalement Inga I et II, il ne suffit pas seulement de coopérer avec la Banque Mondiale, le FMI, avec les Etats étrangers ou initier des projets à vocation régionale, il faut aussi trouver des ressources à l'intérieur de la RDC :

Depuis quelques années, la demande mondiale des métaux non ferreux est tellement forte que des traders, parmi lesquels Glencore, ne pouvaient qu'investir dans les mines en RDC surtout que la paix s'installe. Mais pour assurer la production et le raffinage de cuivre et du cobalt, il fallait aussi de l'énergie, qui est principalement fournie par la SNEL. En décembre 2005, face à la demande pressante d'énergie estimée à 2000 MW à l'horizon 2020, la SNEL a convoqué une table ronde à Lubumbashi, l'ADG a dit aux miniers que la seule façon de résoudre le problème d'énergie était leur implication dans la réhabilitation et la modernisation des équipements de la SNEL sous forme de partenariat public-privé. C'est ce schéma - là qui est en cours de réalisation avec quelques sociétés minières de Kolwezi dont KCC, MUMI et KANKUKI.

Ces trois sociétés ont monté un financement de 300 millions de dollars pour le projet FRIPT (Fiabilisation, réhabilitation et renforcement des infrastructures SNEL de production d'Inga et Nzilo) et de transport (courant continu et courant alternatif associés) dont la consistance inclut un certain nombre de travaux, dont la fiabilisation pole $\mathrm{n}^{\circ} 2$ de la liaison 
THTCC Inga - Kolwezi; la réalisation des actions urgentes du projet Nzilo; la remise en service du groupe $\mathrm{n}^{\circ} 3$ du barrage Nzilo, etc.

Ce projet est non seulement complémentaire aux projets SAPMP et PMEDE, mais il permet d'optimiser l'exploitation de la ligne THTCC -Inga-Kolwezi à sa pleine capacité installée $1000 \mathrm{MW}$. De ces $1000 \mathrm{MW}, 500 \mathrm{MW}$ sont destinés à l'exportation, tandis que 500 MW sont destinés à la consommation du Katanga (près de 400 MW seront destinés aux industries minières qui ont monté le financement et $100 \mathrm{MW}$ seulement seront à la disposition de la SNEL pour la population) ${ }^{19}$.

Questions juridiques y relatives;

Il convient de souligner que des questions juridiques en rapport avec ces projets de modernisation et d'extension d'Inga I et II se posent. Notamment les caractères non commutatif et opaque de tous ces contrats que la république signe avec les IFI pour la réalisation de ces projets. Parce que ce qui est révéler au public c'est de dire que la Banque mondiale a accordé un don de X montant à la RDC pour le secteur de l'électricité, mais en réalité ce ne sont pas de dons mais des contrats de prêt. Le constant est que beaucoup de ces contrats sont léonins et ceci fait à ce que la RDC s'enfonce dans l'endentement. Et la satisfaction des besoins en électricité du pays reste une question qui est loin d'être résolue en dépit de ces multiples projets. Aussi, les problèmes de responsabilité et d'inexécution des marchés publics accordés à certaines entreprises concessionnaires du secteur. C'est pourquoi, il faut au préalable bien définir les obligations des parties en présence, définir le cahier des charges et renforcer le contrôle tant parlementaire que juridictionnel afin d'établir la transparence des contrats conclus par la république et la responsabilité de la partie défaillante.

\section{$3^{\circ}$. DE LA MODERNISATION DU GRAND INGA}

Faisabilité :

A l'origine, selon le plan directeur de la SNEL, l'aménagement du Grand Inga prévoit en amont la construction d'un premier barrage long de $630 \mathrm{~m}$ et haut de $140 \mathrm{~m}$, censé détourner le cours du fleuve vers la vallée asséchée de la Mbundi, d'un second; le barrage de Sikila (850 m de long et $70 \mathrm{~m}$ de haut); d'une digue, longue de $550 \mathrm{~m}$ et haute de $45 \mathrm{~m}$, enfin, la construction du barrage en enrochement de la Mbundi $(1600 \mathrm{~m}$ de long et $160 \mathrm{~m}$ de haut) où seraient localisées les 52 turbines de $750 \mathrm{MW}^{20}$.

Pour la mise en œuvre de ce méga projet, un consortium géant réunit la RDC, l'Afrique du Sud, l'Espagne et la Chine, sous l'égide de la Banque mondiale(BM), la Banque afri-

19 KAUKI Faustin, Trois entreprises minières du Katanga prêtes à coopérer avec la Banque Mondiale pour fiabiliser la ligne à haute tension Inga-Kolwezi, in digital.congo.net, Kinshasa 05/07/2012.

20 SNEL, Plan directeur horizon 2015, janvier 2005. 
caine de développement(BAD) et la Banque européenne d'investissement(BEI). Les firmes EDF et GDF sont associées au projet pour tout ce qui concerne l'ingénierie.

Lors du sommet de Cannes en Novembre 2011, le G20 avait fait de Grand Inga un des projets phares de son agenda développement. De même, lors du sommet sur « l'Energie durable pour tous » du 16 avril 2012 organisé à Bruxelles par l'Union Européenne et l'ONU, le vice-président de la BAD pour les infrastructures, Gilbert Mbesherubusa a annoncé la décision de construire étapes par étape le barrage de Grand Inga ${ }^{21}$. Prochain épisode : le lancement d'une étude de faisabilité.

De même, le 12 novembre 2011, en présence des Présidents Joseph Kabila de la RDC et Jacob Zuma d'Afrique du Sud, les ministres de l'énergie de deux pays avaient posés les jalons en signant un protocole d'accord pour la construction du Grand Inga.

Grand Inga est aussi listé comme projet prioritaire de la SADC, du NEPAD et du Conseil Mondial de l'Energie. C'est assurément son grand potentiel de rentabilité qui motive l'intérêt de nombreuses structures : Banque Mondiale, Banque Africaine de Développement, Banque Européenne d'Investissement, Banques Privées occidentales, Organismes régionaux, Multinationales..., pour le financement de ce projet qui vanécessiter de milliards de dollars au bas mot.

D'une capacité de 40.000 MW (40 GW) ce monstre béton et les ouvrages associés devraient générer une puissance supérieure à celle combinée de deux plus grands barrages existants du monde : celui des Trois Georges sur le Yangzi Jiang en Chine (22,5 GW) et celui d'Itaipu (14 GW) sur le Rio Paraná, à la frontière du Brésil et du Paraguay.

Un projet pharaonique : le coût de barrage et des autoroutes de l'énergie, des lignes à très haute tension censées acheminer le courant d'Inga aux quatre coins du continent, voire en Europe et au Moyen Orient, estimé à plus de 80 milliards de dollars, dans un continent où plus de 900 millions de personnes sont privées d'électricité.

L'ouvrage a de quoi impressionner. Même les ingénieurs chinois blasés par la présence en leur pays de l'immense barrage de Trois-Georges, s'inclinent devant le gigantisme du projet Grand Inga ${ }^{22}$.

Précisons que la modernisation du Grand d'Inga, va débuter avec sa première partie qui est Inga III. Selon le gouvernement de la RDC, Inga III nécessite une enveloppe globale de 12 milliard de dollars. Selon les études de préfaisabilité disponibles au ministère des ressources hydrauliques et électricité, la centrale aura une puissance installée de 4800 MW, soit deux fois plus que la puissance des centrales Inga I et II réunies. La hauteur de la chute sera de 70 mètres, les conduites d'aménagées d'eaux auront 13 mètres de diamètre et une longueur de 6700 mètres, avec 16 tunnels. Son débit sera de $6600 \mathrm{~m}^{3}$ par seconde. Il devrait donc compléter ce dispositif existant. Ainsi, Inga III permettra d'augmenter les exportations d'électricité jusqu'à $20 \mathrm{TW}$ h, contre $2 \mathrm{TW}$ h actuellement. Il pourra générer des revenus

21 MISSER François, Le Barrage de Grand Inga, victime de changement climatique, in www.gloogle.fr (7/04/2013).

22 PNUE, op.cit, p. 7. 
annuels de plus de 1 milliard de dollars. Et pour Bruno Kapanji, le projet Inga III n'est qu'une phase de Grand Inga ${ }^{23}$. La RDC a fixé à octobre 2015 le début des travaux sur les rives du fleuve Congo.

Et donc les extensions envisagées, pourront transformer le site en un fournisseur d'énergie important à l'échelle globale.

Il revient donc à la République avec ses partenaires susvisés d'insérer dans leur partenariat des conditions juridiques qui soient bénéfiques tant à la population congolaise qu'aux investisseurs. Il s'agit des conditions juridiques commutatives et équilibrées.

Enfin, parce qu'il faut des conditions nécessaires, déjà, la RDC et l'Afrique du Sud étaient en discussion pour la signature d'un traité de mise en œuvre d'un plan de construction de Grand Inga, par suite de la signature d'un protocole d'accord entre les 2 pays en 2011. Et que finalement le projet de Traité de coopération énergétique a été signé 2013.

\section{DU TRAITE DE COOPERATION ENERGETIQUE SUR LE PROJET DE CONSTRUCTION DE GRAND INGA}

Bruno Kapandji Kalala et Bikobe Ben Martin's, respectivement ministres en charge de l'électricité de la RDC et la République Sud Africaine, ont signé le 30 octobre 2013 à Kinshasa le Traité de coopération énergétique sur le projet de construction de Grand Inga, sur le site Inga au Bas-Congo.

Ce Traité fait suite au Protocole d'accord entre les deux pays le 12 novembre 2011 à Lubumbashi, qui a été matérialisé par le Projet de Traité paraphé par les deux ministres également à Lubumbashi, le 7 mars 2013.

Ce Traité énonce en substance les conditions juridiques suivantes :

- La propriété de la RDC sur le site d'Inga et son leadership sur le développement de 8 différentes phases du site d'Inga à commencer à Inga III;

- Le caractère régional africain du projet;

- La transparence dans toutes les opérations de préparation, de financement, de passation des marchés, de construction et d'exploitation des infrastructures de production et de transport d'énergie,

- Le développement en mode partenariat public-privé avec tous les partenaires intéressés au projet;

- Concernant Inga III ou phase1 du projet Grand Inga, ce Traité confirme entre autre l'engagement de la RSA à acheter $2500 \mathrm{MW}$ sur les $4800 \mathrm{MW}$ qui seront produits. Ce qui rend le projet Inga III, en cours de préparation, bancable et crédible. Il est bien sur les rails pour sa réalisation prévue à l'horizon 2020;

- La durée du Traité est de 10 ans;

23 BRUNO KAPANJI, Ministre des Ressources hydrauliques et Electricité, in www.radiookapi.net (3/04/2014.). 
- Un accord formel d'achat sera négocié et signé en temps opportun entre les sociétés nationales d'électricité de ces deux pays ${ }^{24}$.

Dans le cadre de suivi de mise en œuvre des engagements des parties, les deux parties s'engagent à mettre en place :

- La commission ministérielle conjointe(CMC) qui sera composée des ministres en charge de l'électricité de chaque Etat et de tout autre ministre dont la présence est jugée nécessaire,

- La commission technique permanente(JPTC) qui sera composée d'experts de deux Etats en vue de faciliter la mise en œuvre du Traité.

La dernière réunion technique tenue entre la partie congolaise et sud africaine à Pretoria du 5 au 9 février 2014 apporte la preuve supplémentaire de l'intérêt de différents partenaires à la réalisation du projet Inga III. Bernard Dayayele (coordonnateur de la cellule de gestion Inga III) témoigne sa sérénité à la fin des travaux des experts. La réunion de Pretoria constitue une étape importante dans la préparation d'une feuille de route balisant le chemin aux différentes recommandations des chefs d'Etats et devant aboutir à la signature d'un Traité commercial pour l'exploitation du barrage Inga III.

A la tête de la délégation des experts congolais, le ministre des Ressources hydrauliques et électricité, Bruno Kapandji, a séjourné en Afrique du sud pendant 5 jours dans le cadre du processus de modernisation du projet Grand Inga. Aux termes des travaux, les 2 personnalités ont paraphé l'accord énergétique entre la RDC et la RSA.

Comme on le voit, d'autres traités, accords et contrats de partenariat seront conclus toujours dans le cadre de renforcer des conditions juridiques nécessaires à la modernisation et extension d'Inga.

\section{§.2. DES CRITIQUES ET SUGGESTIONS AUX PROJETS}

\section{HYDROELECTRIQUES D'INGA}

Comme nous l'avons dit, pour moderniser et étendre le complexe hydroélectrique d'Inga; il faut mobiliser des ressources financières importantes afin de procéder à ces projets à vocation régionale et internationale. Ces projets font face à une myriade de défis politique, techniques, financiers et environnementaux. Et si les conditions juridiques de modernisation ne sont pas équitables (gagnant-gagnant), l'inquiétude demeurerait de voir la RDC replonger dans la spirale de l'endettement enclenchée par la construction des barrages existants (Inga I et II) et par la crainte qu'à nouveau les congolais restent dans l'obscurité aux pieds des pylônes des lignes exportant ailleurs l'électricité d'Inga. Il se pose ici la question de la réalité, de la rentabilité et de responsabilité de ces projets.

Ainsi, la mise en œuvre de ces conditions juridiques et lecontrôle de la transparence des projets et contrats susvisés ne peuvent être efficaces dans un Etat qui se veut de droit

24 Traité de coopération énergétique entre RDC et RSA, Kinshasa, 30/10/2013. 
comme la RDC, que par une bonne gouvernance de la chose publique par le contrôle parlementaire mais aussi par le contrôle juridictionnel.

De plus, la mise en place d'Inga III et Grand Inga entrainera une avalanche de nuisances économiques graves (rejets de grandes quantités des produits toxiques dans l'eau et les sols, migration des poissons...) et des déplacements forcés de populations.

Aussi une hypothèque pèse sur le projet Grand Inga : celle du changement climatique entrainant la sécheresse, phénomène qui a des conséquences fâcheuses sur le débit du fleuve Congo. ${ }^{25}$

C'est pourquoi les sponsors du concept Grand Inga devraient initier au préalable une évaluation stratégique sociale et environnementale le plus tôt possible, de façon à ce que les problèmes majeurs soient traités en phase initiale de planification. Cette évaluation devrait inclure des questions environnementales telles que le barrage lui-même, les impacts en aval et en amont du barrage en étendant l'étude à l'embouchure et au-delà le tracé des lignes de transmission, et les questions sociales comme l'impact sur la migration, l'emploi et le développement qui dépendent des options de distribution d'énergie électrique. Bref, la réponse à la question de responsabilité de l'Etat, de ses partenaires ou des entreprises du secteur face aux besoins des populations locales, tel que mentionner dans la loi sur la conservation de la nature ${ }^{26}$ et la loi sur le secteur de l'électricité.

\section{CONCLUSION}

Le rôle et l'importance de la modernisation et l'extension du site d'Inga dans la politique énergétique de la RDC sont cruciaux. Le développement de ce complexe est un atout majeur pour l'économie congolaise et un facteur d'intégration régionale.

Ce projet nécessite la mobilisation d'importantes ressources financières qui demande un engagement fort de l'Etat congolais et autres Etats partenaires. Mais aussi la participation du secteur privé.

Cette modernisation vise d'une part la réhabilitation des barrages existants d'Inga I et II pour faire face au déficit énergétique et l'approvisionnement de grands consommateurs et l'export d'électricité vers le continent. Il ressort que même avec cette réhabilitation d'Inga, le déficit serait encore croissant en RDC à l'horizon 2020. Ainsi, d'autre part, il est nécessaire d'entreprendre le développement de nouveaux projets sur Inga dont le Grand Inga, à dimension continentale, voire mondiale.

Les conditions juridiques nécessaires à cette modernisation s'expriment d'une part par l'existence d'une loi cohérente, attractive et adaptée à l'évolution du secteur de l'électricité et d'autre part par des règles de partenariat public-privé et des conventions ou traités internationaux qui détermineront l'engagement politique et économique de diverses parties pre-

25 Lire à ce sujet les Rapports du PNUE susvisé et du Forum du Ministère des Ressources Hydrauliques et Electricité, sur l'étiage du fleuve Congo et son impact sur la fourniture de l'énergie, Kinshasa, juin2012.

26 Articles 9 à 18 de la Loi $N^{\circ} 14 / 003$ du 11 Février 2014 relative à la conservation de la nature. 
nantes à ces projets. Il est nécessaire de prendre en compte dans ces conditions juridiques les expériences de partenariat public-privé réussi en Afrique et dans le monde et d'adopter les meilleurs pratiques internationales de montage des projets.

A ce sujet, la loi sur le secteur de l'électricité en RDC a été adoptée le 15 novembre 2013. Elle est donc sur la table du président de la république pour sa promulgation. Aussi, la RDC et l'Afrique du Sud ont signé le 7 mars 2013 un Traité de coopération énergétique pour la mise en œuvre de la modernisation du Grand Inga.

\section{NOTES BIBLIOGRAPHIQUES}

1. Constitution de la $\mathrm{RDC}, 18 / 02 / 2006$, telle que révisée à ce jour.

2. Traité de coopération énergétique entre RDC et RSA, Kinshasa, 30 0ctobre, 2013;

3. Loi $N^{\circ} 14 / 003$ du 11 Février 2014 relative à la conservation de la nature, Journal officiel, RDC, 2014;

4. Loi relative au secteur de l'électricité en RDC, Cabinet du Premier Ministre, novembre 2013;

5. Ordonnance-loi $n^{\circ} 70 / 033$ du 16 mai 1970 portant création de la SNEL;

6. KASEMUANA Séraphin, Rapport : Energie et écodéveloppement en RDC, Hélio International, Observatoire de la viabilité énergétique, Kinshasa, 2006;

7. KINTAMBU KIBANIMBA, Estimation des besoins et coûts dans le secteur de l'énergie électrique pour l'atteinte des OMD dans le cadre d'une planification axée sur les résultats, Mémoire, Economie et Finance, UNIKIN, Kinshasa, 2009;

8. LUKOMBE NGHENDA, Zaïrianisation, Radicalisation et Rétrocession en République du Zaïre, Considérations juridiques, éd. PUZ, Kinshasa, 1979;

9. MUKADI José, Historique de la mise en route du barrage d'Inga, Kinshasa, 2006;

10. RUDO SANYANGA, Barrage Grand Inga, $R D C$, in International Rivers, Berkeley, USA, 2012;

11. RDC/AFD : Agence Française de développement et le barrage Inga III, Kinshasa, juin 2012;

12. Ministère de l'Energie, Rapport du colloque annuel des ministres de l'énergie de la SADC, Kinshasa, juin 2001;

13. Ministre des Ressources hydrauliques et Electricité, Forum sur «le Fleuve Congo et son impact sur la fourniture de l'énergie, Kinshasa, juin, 2012;

14. MISSER François, le barrage de Grand Inga victime de changement climatique, in www.gloogle.fr;

15. PNUE, Rapport sur le projet hydroélectrique d'Inga, Kinshasa, septembre, 2011;

16. SNEL, Plan directeur horizon 2015, Lubumbashi, 2005;

17. Revue du DSCRP, RDC, Kinshasa, mars 2010;

18. TOUROT Nicolas, Les Projets de développement hydroélectrique en Afrique Noire, Mémoire de maîtrise, Paris I, 2003;

19. VAN DEUREN Pierre, Aménagement du Bas-Congo, Projet, Bruxelles, 1928; 
DES CONDITIONS JURIDIQUES NECESSAIRES A LA MODERNISATION ET A L'EXTENSION DU BARRAGE HYDRAULIQUE D'INGA.

20. YUMA MULIMBI Albert,Le rôle du secteur privé congolais dans la reconstruction de l'Etat et la réalisation d'un développement économique durable en $R D C$, in Conférences FEC, Bruxelles, 2008;

21. www.radiookapi.net (O8/04/2014);

22. www.banquemondiale.org (09/04/2014);

23. www.bhpilliton.com $(11 / 12 / 2013)$;

24. www.sapp.co.zw $(12 / 02 / 2014)$;

25. www.Snel.cd (09/04/2014). 